University of Nebraska - Lincoln

DigitalCommons@University of Nebraska - Lincoln

2012

\title{
Response of spherical diffusion flames subjected to rotation: Microgravity experimentation and computational simulation
}

\author{
Sean W. Yoo \\ Princeton University \\ Swetaprovo Chaudhuri \\ Princeton University, sweto@princeton.edu \\ Kurt R. Sacksteder \\ NASA Glenn Research Center \\ Peng Zhang \\ Princeton University \\ Delin Zhu \\ Princeton University \\ See next page for additional authors
}

Follow this and additional works at: https://digitalcommons.unl.edu/nasapub

Part of the Physical Sciences and Mathematics Commons

Yoo, Sean W.; Chaudhuri, Swetaprovo; Sacksteder, Kurt R.; Zhang, Peng; Zhu, Delin; and Law, Chung K., "Response of spherical diffusion flames subjected to rotation: Microgravity experimentation and computational simulation" (2012). NASA Publications. 102.

https://digitalcommons.unl.edu/nasapub/102

This Article is brought to you for free and open access by the National Aeronautics and Space Administration at DigitalCommons@University of Nebraska - Lincoln. It has been accepted for inclusion in NASA Publications by an authorized administrator of DigitalCommons@University of Nebraska - Lincoln. 


\section{Authors}

Sean W. Yoo, Swetaprovo Chaudhuri, Kurt R. Sacksteder, Peng Zhang, Delin Zhu, and Chung K. Law 


\title{
Response of spherical diffusion flames subjected to rotation: Microgravity experimentation and computational simulation
}

\author{
Sean W. Yoo ${ }^{\mathrm{a}, 1}$, Swetaprovo Chaudhuri ${ }^{\mathrm{a}, *}$, Kurt R. Sacksteder ${ }^{\mathrm{b}}$, Peng Zhang ${ }^{\mathrm{a}}$, Delin Zhu ${ }^{\mathrm{a}}$, Chung K. Law ${ }^{\mathrm{a}}$ \\ a Department of Mechanical and Aerospace Engineering, Princeton University, Princeton, NJ 08544, USA \\ ${ }^{\mathrm{b}}$ NASA Glenn Research Center, Cleveland, OH 44135, USA
}

\section{A R T I C L E I N F O}

\section{Article history:}

Received 27 April 2011

Received in revised form 21 July 2011

Accepted 21 July 2011

Available online 19 August 2011

\section{Keyword:}

Rotating spherical diffusion flame

\begin{abstract}
A B S T R A C T
Microgravity experiments were conducted in the 2.2-s drop tower and zero-gravity facility at NASA-GRC to gain fundamental understanding of the effects of spinning on an otherwise spherical diffusion flame. The flames were generated by injecting either a fuel or an oxidizer mixture from a porous burner to a controlled ambient of either an oxidizer or fuel mixture, respectively. Results show that the polar flame location scales with the angular velocity monotonically as $\omega^{a}$, where $a$ is greater and smaller than unity for small and large spinning velocities, respectively. On the contrary, the equatorial flame location responds nonmonotonically to increasing spinning velocity: first increasing and then decreasing. The experimental observations agree well with the computational simulation where the simulated results demonstrate that the nonmonotonic response of the equatorial flame location is caused by dilution of the reactant concentration in the outwardly-directed radial flow by the product and inert that are carried by the inwardly-directed polar flow upon traversing the flame segment in the polar region.
\end{abstract}

(c) 2011 The Combustion Institute. Published by Elsevier Inc. All rights reserved.

\section{Introduction}

The phenomenon of spherical flame subjected to a spinning flow is of both practical and fundamental interest, particularly when the fuel is in the condensed phase such that spherical symmetry of the fuel source is maintained. Practically, we note as examples that injected fuel droplets and particles frequently acquire a spinning motion upon either injection or being acted on by the vortical motion of the turbulent eddies. Fundamentally, while considerable research has been performed on burning droplets and particles in rectilinear motion, studies on effects of spinning motion have been relatively few, leading to uncertainty in our ability to describe such relevant issues as, say, the relative roles of fluid entrainment at the pole region and discharge in the equator region on the flame response, and the coupling between spinning and the gasification process.

In terms of prior work, Pearlman and Sohrab [1] experimentally found that rotation enhances the vaporization rate of a droplet. Similar conclusions were reached by Lozinski and Matalon [2,3], who analyzed pure vaporization [2] and burning from a spinning fuel droplet with unity Lewis number, Le [3]. Yoo et al. [4] extended the theoretical investigation to include general Lewis numbers, and found that $L e$ indeed can significantly modify the response of the

\footnotetext{
* Corresponding author.

E-mail address: sweto@princeton.edu (S. Chaudhuri).

${ }^{1}$ Present address: GE Aviation, Seattle, WA 98124, USA.
}

rotating flame. Specifically, it was shown that for a moderate range of the ambient oxygen mass fraction, the flame would experience a temperature reduction at the poles but an elevation at the equator when $L e_{\mathrm{F}}>1$ or $L e_{\mathrm{O}}<1$, while the response is reversed for $L e_{\mathrm{F}}<1$ or $L e_{\mathrm{O}}>1$.

A parallel perturbation study has also been conducted for a burner-generated premixed flame under rotation [5]. Being a premixed flame and hence possessing a distinctive flame speed that fundamentally depends on the coupled influence of the mixture $L e$ and the nature of stretch, the response of the flame to the rotating flow can be substantially different from that of the diffusion flame. For example, instead of always having the flattened surface at the poles for diffusion flames, flattening can occur at either the pole or equator for premixed flames.

The richness of the theoretical predictions [2,3] necessitates correspondingly well-controlled experimentation, for verification as well as extension. In particular, it is recognized that since the analyses were only for small rotational velocities, experimentation with larger rotational velocities should reveal flame response not accessible to the perturbation analysis. Furthermore, the distinctively different response of the diffusion flame from the premixed flame under rotation should be easily verified experimentally.

In response to the above considerations, a systematic experimental investigation of the effects of rotation on a burner-generated spherical diffusion flame in a well-controlled microgravity environment was undertaken. Unlike the droplet flame, the mass flux from the burner is prescribed so the flame standoff distance 
can be easily manipulated. Furthermore, one can readily control the $L e$ of the burner and the ambient gases by diluting the respective reactants with inerts of different diffusive properties. Three sets of experiments were chosen with different Le and fuel/oxidizer combination as burner or ambient fluid to investigate the flame dynamics at high angular velocities. While it was found that the polar displacement scales approximately as that of a spinning disk, an interesting phenomenon was observed in the equatorial flames - namely the nonmonotonicity of the equatorial flame location with respect to increasing rotational velocity. We have subsequently conducted a parallel computational simulation which satisfactorily identified the cause for such a phenomenon and, through it, obtained useful insight into the flame response for the general class of flames subjected to flow entrainment.

\section{Experimental apparatus}

The microgravity experiments were conducted in the 2.2-s drop-tower and the 5.18-s Zero-G research facility at the NASA Glenn Research Center. In this paper only results from the experiments at 2.2-s drop tower are presented while those from the Zero$\mathrm{G}$ facility are given in Yoo [6].

\subsection{Combustion drop rigs}

The microgravity experiments were conducted using two drop rigs. The "PIG I Arnold" was equipped with a windowed pressurized vessel, and was used for cases that required the ambient to comprise of reactants other than standard air. For flames that were established in normal air, the Princeton Drop Rig (PDR) with the combustion chamber open to the atmosphere was used. The design is otherwise similar to that of the pressurized rig. Figure 1a shows the spinning combustion module inside the pressure vessel, with the electronics and flow system integrated to the rig. The spherical, porous, burner $(0.95 \mathrm{~cm}$ in diameter), with pore size ranging from 7 to $15 \mu \mathrm{m}$, was fabricated in our laboratory by sintering bronze powders (diameter ranges from 5 to $13 \mu \mathrm{m}$ ) inside a custom-made graphite mold, which was heated in a well-controlled, high-temperature oven [7]. The sphere is supported and fed by a stainlesssteel capillary, which has an O.D. of $1.1 \mathrm{~mm}$ and an I.D. of $0.08 \mathrm{~mm}$. Although it is not apparent in Fig. 1a, a stationary, outer stainless steel capillary ( $1.5 \mathrm{~mm}$ O.D., $1.3 \mathrm{~mm}$ I.D.) is installed around the inner tube to suppress the rotational motion by the inner support capillary. The rotational speed of the burner was controlled by a variable speed DC servo motor that can spin up to $1800 \mathrm{rpm}$. Details of both rigs and specifications of the fuel delivery system are given in [6].

\subsection{Ignition and diagnostics}

Since microgravity laminar flames are sensitive to hydrodynamic disturbances, especially those caused by the flame and the buoyant plume of the product gases released by ignition at normal gravity, design and control of the ignition procedure were critical in obtaining useful data. The time between the release of the burner gas and actual ignition was optimized and controlled by a serial pair of solenoid valves such that enough time was allotted for the flow system to reach steady state and that enough premixture is present before the ignition. The total amount of the burner gas released before ignition, however, was minimized to prevent the formation of a large unsteady, premixed flame. The flame was ignited immediately after release of the rig by energizing a $\mathrm{Ni}$ chrome coiled wire that was attached onto a retractable ignition arm. The coil was energized at 28 VDC, $4.5 \mathrm{ohm}$ resistance for $150 \mathrm{~ms}$ with a total energy of $18.9 \mathrm{~J}$ and was immediately retracted
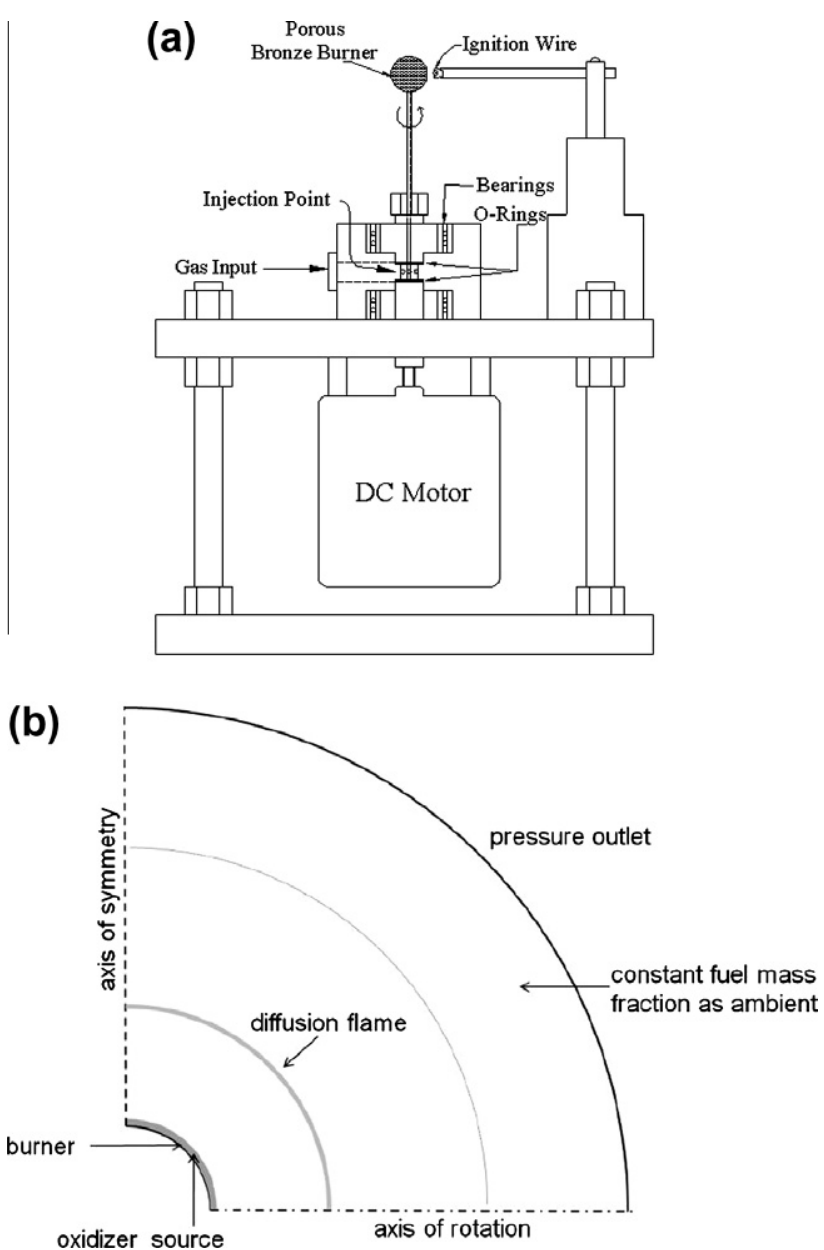

Fig. 1. (a) Schematic of the spinning combustion module and (b) schematic of the computational domain.

by a rotary actuator following ignition. The burner was rotated even before the pre-flow and sufficient time was assigned for the rotating flow field to reach steady state. The volumetric flow rate ranged from 5 to $15 \mathrm{~mL} / \mathrm{s}$. The visible flame image was recorded onto a mini DV cassette with a high-resolution color CCD camera and a more sensitive $\mathrm{B} / \mathrm{W}$ camera. The relative times were encoded onto the video data, recorded at 30 frames per second, with a synchronized time-code generator.

\section{Experimental design}

Three series of experiments were conducted, with their relevant information given in Table 1 . An important consideration in the design of the experimental running conditions is to minimize the heat loss to the burner, which is difficult to quantify. This is accomplished by maximizing the flame-front standoff distance within the experimental constraints, which in turn are affected by the flow system, the design of the porous burner, microgravity time, and the allowable flame size. For example, the mass flow rate or the flame size was limited by the available microgravity time since the flame needed more time to reach steady-state as the steadystate flame size became larger. In addition, if the mass flow rate was made too large, some flames would extinguish due to radiation. Consequently, the reactant concentration in the ambient was minimized while the mass flow rate of the burner mixture was maximized. Most importantly, increasing the mass flow also enhances the perturbation induced by the rotational motion of the burner [4]. 
Table 1

Composition and Le of fuel and oxidizer mixtures.

\begin{tabular}{|c|c|c|c|c|c|c|c|}
\hline & & Composition & $L e_{\text {mixture }}$ & $\mathrm{Le}_{\mathrm{CH}_{4}}$ & $L e_{\mathrm{I}}$ & $\alpha\left(\mathrm{cm}^{2} / \mathrm{s}\right)$ & $m(\mathrm{mg} / \mathrm{s})$ \\
\hline Case 1 & $\begin{array}{l}\text { Burner gas } \\
\text { Ambient gas }\end{array}$ & $\begin{array}{l}21 \% \mathrm{O}_{2} / 79 \% \mathrm{~N}_{2} \\
10 \% \mathrm{C}_{2} \mathrm{H}_{4} / 90 \% \mathrm{Ar}\end{array}$ & $\begin{array}{l}1.10 \\
1.27\end{array}$ & $\begin{array}{l}\text { NA } \\
\text { NA }\end{array}$ & $\begin{array}{l}1.07 \\
1.31\end{array}$ & $\begin{array}{l}0.22 \\
0.19\end{array}$ & $\begin{array}{l}16.87 \\
N A\end{array}$ \\
\hline Case 2 & $\begin{array}{l}\text { Burner gas } \\
\text { Ambient gas }\end{array}$ & $\begin{array}{l}30 \% \mathrm{H}_{2} / 15 \% \mathrm{CH}_{4} / 55 \% \mathrm{~N}_{2} \\
21 \% \mathrm{O}_{2} / 79 \% \mathrm{~N}_{2}\end{array}$ & $\begin{array}{l}0.43 \\
1.10\end{array}$ & $\begin{array}{l}1.53 \\
\mathrm{NA}\end{array}$ & $\begin{array}{l}0.59 \\
1.07\end{array}$ & $\begin{array}{l}0.47 \\
0.22\end{array}$ & $\begin{array}{l}11.20 \\
\mathrm{NA}\end{array}$ \\
\hline Case 3 & $\begin{array}{l}\text { Burner gas } \\
\text { Ambient gas }\end{array}$ & $\begin{array}{l}30 \% \mathrm{O}_{2} / 70 \% \mathrm{~N}_{2} \\
8 \% \mathrm{H}_{2} / 4 \% \mathrm{CH}_{4} / 88 \% \mathrm{~N}_{2}\end{array}$ & $\begin{array}{l}1.12 \\
0.33\end{array}$ & $\begin{array}{l}\text { NA } \\
1.18\end{array}$ & $\begin{array}{l}1.06 \\
0.36\end{array}$ & $\begin{array}{l}0.22 \\
0.29\end{array}$ & $\begin{array}{l}18.70 \\
N A\end{array}$ \\
\hline
\end{tabular}

Each of the fuel and oxidizer for the flame established in case (1) was diluted by an amount of inert with similar thermal diffusivity to attain approximately unity Le. The experimental procedure consists of first determining the optimal mass flow rate by visibly checking the spherical flame dimensions in the absence of rotation. The burner was then rotated and the angular velocity was increased for subsequent drops. The ambient consisted of $\mathrm{C}_{2} \mathrm{H}_{4}$ diluted by $\mathrm{Ar}$ at $0.9 \mathrm{~atm}$, while standard air was introduced through the burner as specified in Table 1 . Although both color and $\mathrm{B} / \mathrm{W}$ videos were recorded, only the $\mathrm{B} / \mathrm{W}$ images are presented here as the color images are not of satisfactory intensity for easy visualization.

For cases (2) and (3), hydrogen was chosen as the fuel for its enhanced ignitability, and methane was added to induce visible emission so that the flame can be detected with regular CCD camera. The ratio of hydrogen to methane was maintained at 2:1. For case (2), the fuel mixture (refer to Table 1 ) was ejected through the burner to the ambient comprised of normal air. Table 1 lists the Lewis numbers computed with mixture averaged diffusivity of the reactant species and that computed with diffusivity relative to the inert, referred as $L e_{\text {mixture }}$ and $L e_{\mathrm{I}}$ respectively. However for most purposes henceforth, only the $L e_{\text {mixture }}$ will be reported, as $L e_{\mathrm{F}}$ or $L e_{\mathrm{O}}$, i.e. Lewis number of the fuel mixture or the oxidizer mixture respectively, unless otherwise mentioned. For the two fuel cases $\left(\mathrm{H}_{2}\right.$ and $\mathrm{CH}_{4}$ in cases 2 and 3$)$, the Le of the fuel mixture $\left(L e_{\mathrm{F}}\right)$ was determined by using hydrogen as the diffusing fuel species, recognizing that it is the dominant fuel species because of its large diffusivity and reactivity. For reference, the Le computed with methane as the diffusing species $\left(L_{\mathrm{CH}_{4}}\right)$ with respect to the mixture is also listed in Table 1.

The running condition for case (3) is similar to that for case (2) except the oxidizer mixture is released from the burner and the fuel comprises the ambient. The oxygen content in the oxidizer stream was increased to $30 \%$ and the fuel in the ambient was heavily diluted with $\mathrm{N}_{2}$, as listed in Table 1 , to match the flame size of case (1) when rotation was not applied. Finally, the flame perturbation with varying mass flow rate $(m)$ will also be investigated, for a fixed, moderate angular velocity of $420 \mathrm{rpm}$.

\subsection{Computational methodology}

The experiment was computationally simulated using the Ansys Fluent software as a two-dimensional axisymmetric problem. The governing equations are given in Ref. [8]. In this code, the pressure-velocity coupling is based on Semi-Implicit-Method for Pressure Linked Equations (SIMPLE) algorithm. The governing equations for $r, \theta, \phi$ momentum, energy and the five species $\left(\mathrm{O}_{2}\right.$, $\mathrm{C}_{2} \mathrm{H}_{4}, \mathrm{~N}_{2}, \mathrm{CO}_{2}, \mathrm{H}_{2} \mathrm{O}$ for case 1 ), were discretized using second-order upwind scheme. A steady solver was used, and mesh independence checked with adaptive meshing implemented based on the gradient of the reaction rate. The schematic of the computational domain is shown in Fig. 1b, in which the burner size with respect to the domain size is greatly exaggerated for ease of visualization. The boundary conditions applied were those of no slip at the bur- ner surface, pressure outlet at the ambient, symmetry along the equatorial radius and axis along the polar radius. To simulate the ambient, a region of constant fuel mass fraction was specified in between the radius which corresponds to the maximum experimental rig dimension and pressure outlet boundary condition which is $\sim 1.5$ times the rig dimension. To simulate the mass flux through the wall, a thin volumetric mass source region given by $\Delta R / R_{\text {burner }}=0.02$ was specified adjacent to the wall, where the thickness $\Delta R$ is much smaller than the boundary layer thickness, which is the smallest possible physical length scale for the rotating cases of interest in this study. The rotation of the burner was simulated by specifying an angular velocity at the burner surface. The main focus of the computational work is to explain the nonmonotonic behavior of the equatorial flame standoff distance at high angular velocities to be described later. One-step global chemistry was used with a reaction rate given by $\omega=B Y_{O_{2}} Y_{F} \exp \left(-E_{a} / R T\right)$, where $\omega=w_{i} /\left[W_{i}\left(v_{i}^{\prime \prime}-v^{\prime}\right)_{i}\right]$ and the nomenclature could be found in [8]. Only case (1) with $\mathrm{C}_{2} \mathrm{H}_{4}$ diluted with $\mathrm{Ar}$ as the fuel and standard air as the oxidizer was simulated; as working with two fuels, i.e. $\mathrm{H}_{2}$ and $\mathrm{CH}_{4}$, as in cases (2) and (3), would require a reaction mechanism that is not well defined within the simplified treatment considered herein, even the phenomenon of interest here is diffusion controlled. Radiation heat loss has not been considered in the current simulations as such effects can be assumed to be of minor importance in governing the flame location for the flame sizes simulated.

\section{Results and discussions}

The flame images at the end of the $2.2 \mathrm{~s}$ drop for cases (1), (2), and (3) at varying angular velocities of the burner are shown in Fig 2. We first note that the quality of the spherical diffusion flames produced by the custom-made, porous bronze burner is good, as evidenced by the largely spherical and smooth flame images in the first column, which has no rotational motion. The bright spots visible in case (2) on the left hand side of the flames are just reflections of the ignition coil, located far behind the flame, on the chamber window.

\subsection{Comparison with theory}

In order to compare with the theory based on the rotational motion being a perturbation to the flow field, the angular velocity needs to be properly non-dimensionalized by $\alpha / l^{2}$ such that the normalized angular velocity is given by $\varpi=\omega \cdot \alpha^{-1} R_{\text {burner }}^{2}[4]$ where $\omega$ is in radians/s, $\alpha$ is the thermal diffusivity of the burner gas and $R_{\text {burner }}$ is the burner radius as the length scale. The $\alpha$ for the three cases are tabulated in Table 1 at the two boundaries. The smallest angular velocity shown in Fig. 2 for case (1) is $80 \mathrm{rpm}$, such that $\varpi=30$, and as such is too large to be considered as a perturbation. Using smaller angular velocities, say $10 \mathrm{rpm}$, steady state would not be reached as the burner would have just made $1 / 3$ of a rotation during the 2.2 -s drop time. 


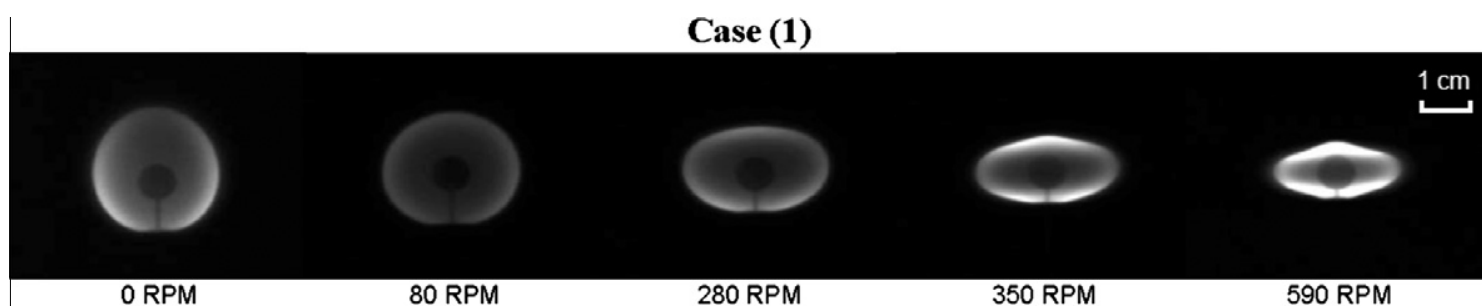

O RPM 80 RPM 280 RPM 350 RPM 590 RPM

Case (2)

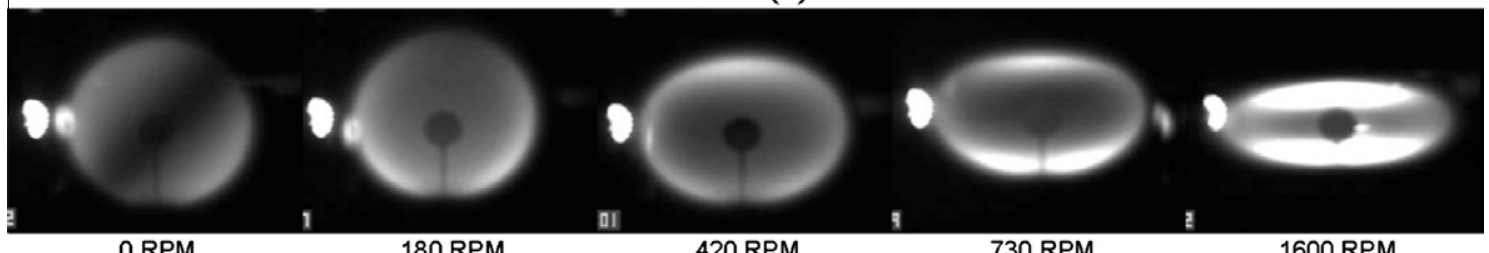

420 RPM

730 RPM

1600 RPM

Case (3)

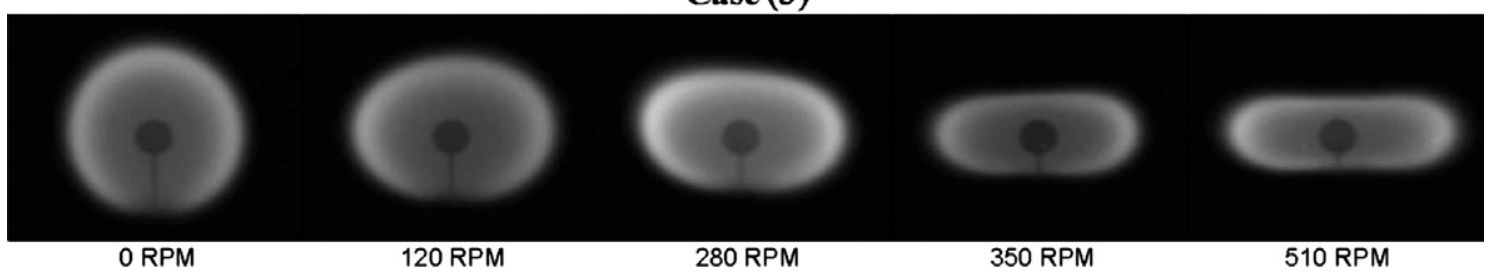

Fig. 2. B/W flame images at the end of $2.2 \mathrm{~s}$ drop as RPM is increased for cases (1), (2), and (3).

Referring to the flame images of Fig. 2, our first observation is that, regardless of the mixture's Lewis number, the otherwise spherical flame is deformed into a pancake shaped flame by the rotational motion, with the flattened surface occurring at the poles. This behavior, while conceptually reasonable and also anticipated from the theory [4], is fundamentally different from the response of premixed flames which depends on the $L e$ of the mixture.

We next note that the flames in cases (1) and (3) seem to respond more sensitively to rotation than in case (2) in that, for a comparable flame deformation, the angular velocity of the burner for cases (1) and (3) are about half that of case (2). This is caused by the different diffusivities of the burner mixture as the angular velocity scales with $\alpha$. Table 1 then shows that the $\alpha$ for case (2) is indeed about twice those of cases (1) and (3).

Thirdly, although not quantitatively obvious from the images shown in Fig 2, the flattening at the poles is greater than the outward protrusion at the equator. This is physically reasonable and also agrees with the theory, which predicts stronger response at the pole by a factor of two as compared to the equator. This factor of two accounts for the increase in the dimension as the onedimensional flow entrained at the poles is driven out to the equator as a two-dimensional flow. Mathematically, for small perturbations the rotation introduces on all perturbed quantities a second Legendre polynomial factor $P_{2}(\cos \phi)=\left(3 \cos ^{2} \phi-1\right) / 2$, which is +1 at the poles and $-1 / 2$ at the equator.

Lastly, there are subtle changes in the distribution of flame emissions between the three cases which demonstrate non-equidiffusive effects. It is observed from Table 1 that all oxidizer mixtures in this study have $L e_{\mathrm{O}} \sim 1$, i.e. always equidiffusive. Thus the non-equidiffusive effects due to changes in curvature, by protrusion or flattening, are restricted to the fuel side only and such effects are expected to be analogous to that of a premixed flame. For case $(1), L e_{\mathrm{F}}=1.27$. Note that the curvature $\kappa$ is positive with respect to the fuel side, with the convention that the curvature is positive when it is convex towards fuel side. With increasing rotation the polar region is flattened thus $\kappa_{\text {polar }}$ is reduced. This reduces

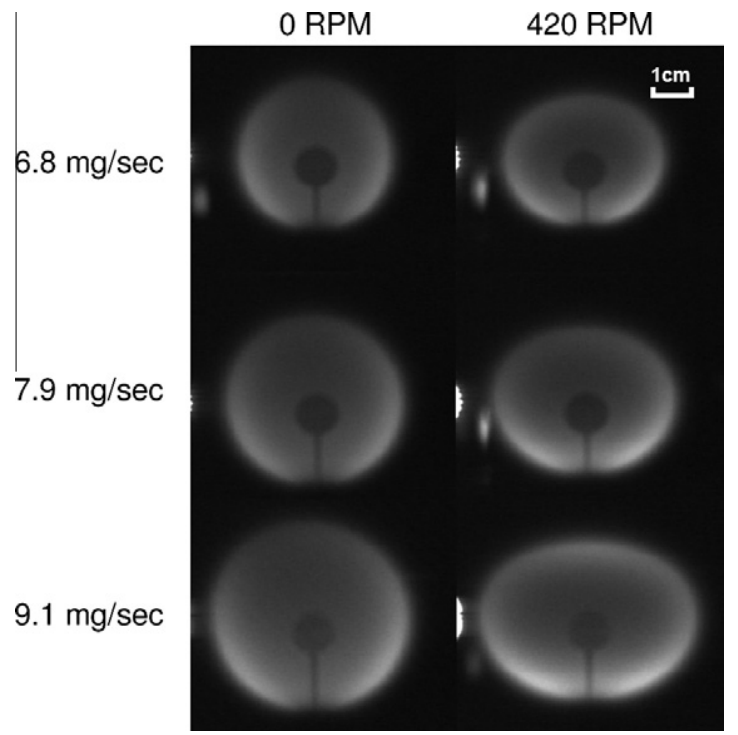

Fig. 3. B/W flame images at the end of the $2.2 \mathrm{~s}$ drop for mass flow rates 5.6, 9.1, and $11.2 \mathrm{mg} / \mathrm{s}$ with and without rotation.

the heat loss and increases the flame temperature and chemiluminescence in the polar region, as shown in Fig. 2. For case (2), $L e_{\mathrm{F}}=0.43$ and $\kappa$ is negative for no rotation. Thus the local temperature is increased as $\kappa_{\text {polar }}$ becomes less negative with increasing rotation. Furthermore, Case (3) has $L e_{\mathrm{F}}=0.33$ but positive $\kappa$, hence the polar temperature and emission are reduced with rotation. For the same reason, exactly the opposite happens for the equatorial location as flattening at poles is accompanied by protrusion at the equator. These finding are in accordance with theoretical predictions of [4].

Images of the flames generated for the three mass flow rates with the same angular velocity (Fig. 3) show that, with increasing 


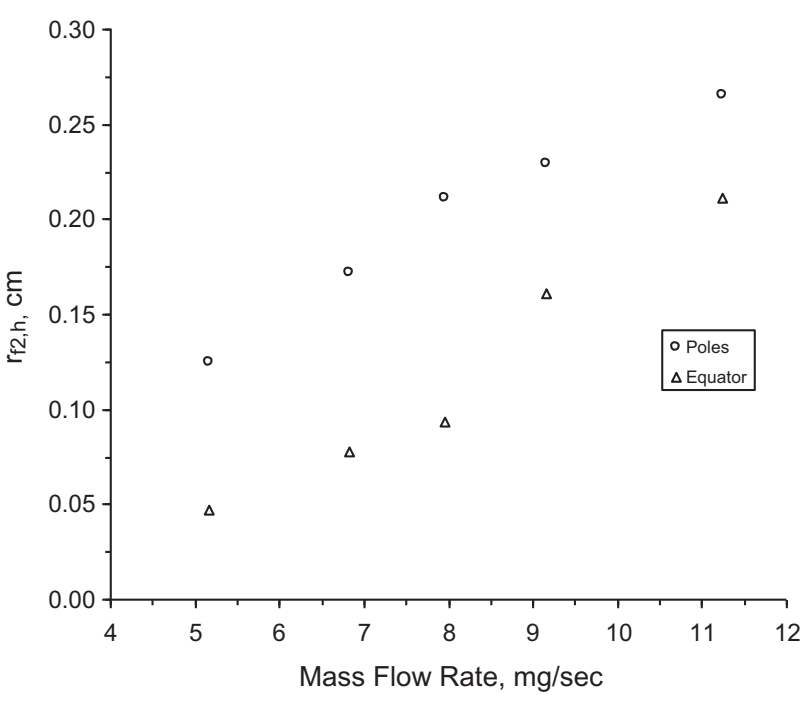

Fig. 4. Plot of $r_{\mathrm{f} 2 \mathrm{~h}}$ versus mass flow rate, where $r_{\mathrm{f} 2 \mathrm{~h}}$ is the difference between the radius of the spherical diffusion flame and the major axis of a flame with rotation of 420 RPM.

mass flow rate their unperturbed size increases, as expected, while the extent of the deformation also increases. This is shown in Fig. 4, in which the flame shape perturbation at the equator/poles, defined as the difference between the radius of the spherical flame and the major/minor axis of deformed flame, is plotted versus $m$. These results suggest that, even at $420 \mathrm{rpm}$, or $\varpi=21$, the flame perturbation still agrees with the perturbation theory [4] and increases linearly with $m$.

\subsection{Scaling analysis of flame shape deformation}

In order to quantify how the flame shape changes with rotation, a series of additional experiments were conducted to determine the extent of the flame deformation with varying angular velocities for a fixed mass flow rate. Since many microgravity experiments were required for this study, the flame that required minimal preparation and was relatively easy to ignite were chosen, which was that specified previously as case (2). Hence similar experiments were conducted for case (2) at two additional mass flow rates: 5.6 and $8.4 \mathrm{mg} / \mathrm{s}$. Each drop was repeated at least twice and the cases that yielded the best symmetric flames with minimal external perturbations were selected for the scaling analysis.

In Fig. 5a and b, images taken at the end of $2.2 \mathrm{~s}$ drops are shown as the magnitude of rotation increased for flames established by issuing a fuel mixture at 5.6 and $8.4 \mathrm{mg} / \mathrm{s}$, respectively. The

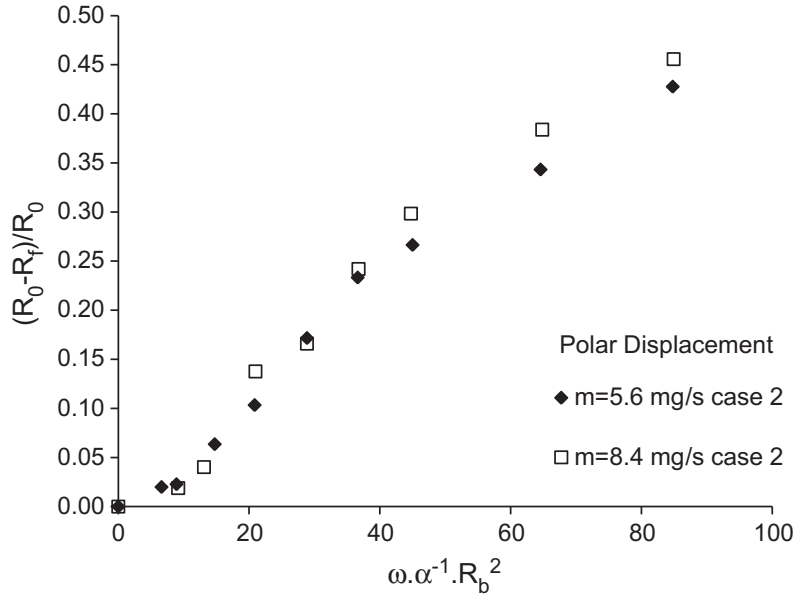

Fig. 6. Plot of flame displacement at the poles versus angular velocity of the burner for flames established under the same condition as case (2) with mass flow rates: 5.6 and $8.4 \mathrm{mg} / \mathrm{s}$.

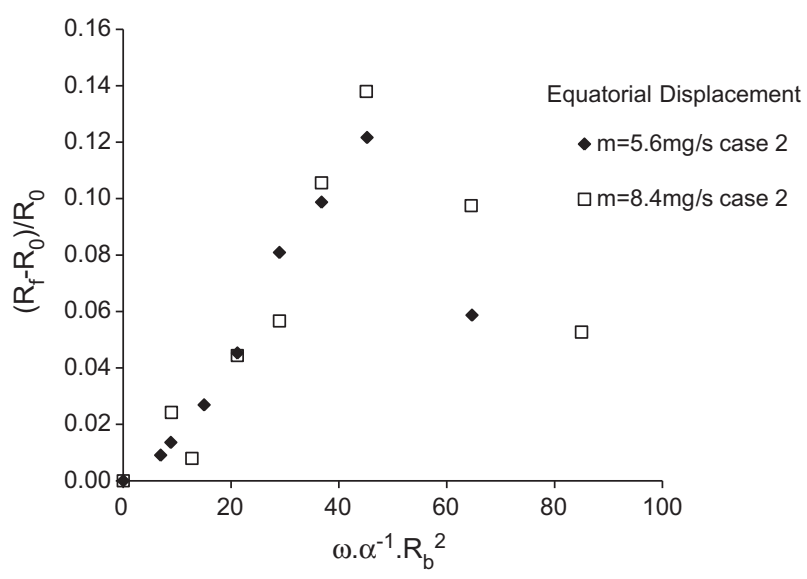

Fig. 7. Plot of flame displacement at the equator versus angular velocity of the burner for flames established under the same condition as case (2) with mass flow rates: 5.6 and $8.4 \mathrm{mg} / \mathrm{s}$.

responses of these flames are similar to that shown in Fig. 2 for case (2), except that the flames are smaller. It is seen that the flame flattens at the poles and protrudes around the equator with increasing angular velocities. However, when the normalized angular velocity becomes sufficiently large $(\varpi>50)$, the flame no longer protrudes around the equator and begins to retract. If the rotational velocity is increased further, beyond $\varpi=85$, the flame will eventually extinguish at the two poles due to excessive heat loss.

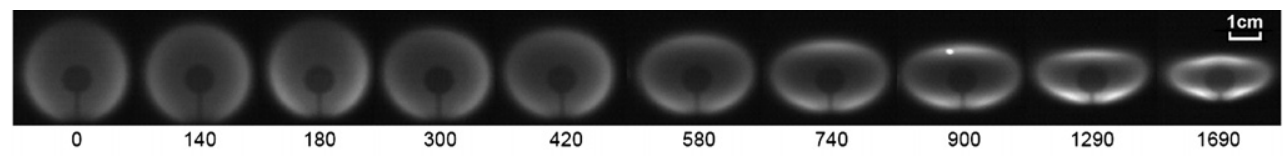

(a)

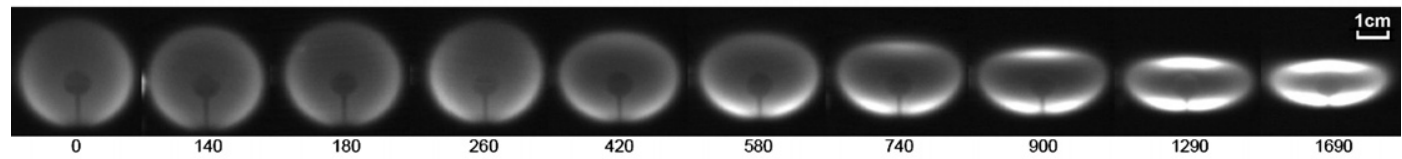

(b)

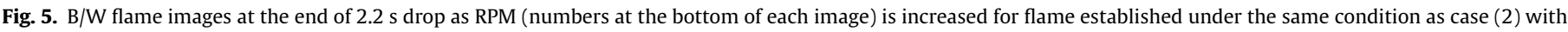
mass flow rates: (a) $5.6 \mathrm{mg} / \mathrm{s}$ and (b) $8.4 \mathrm{mg} / \mathrm{s}$. 


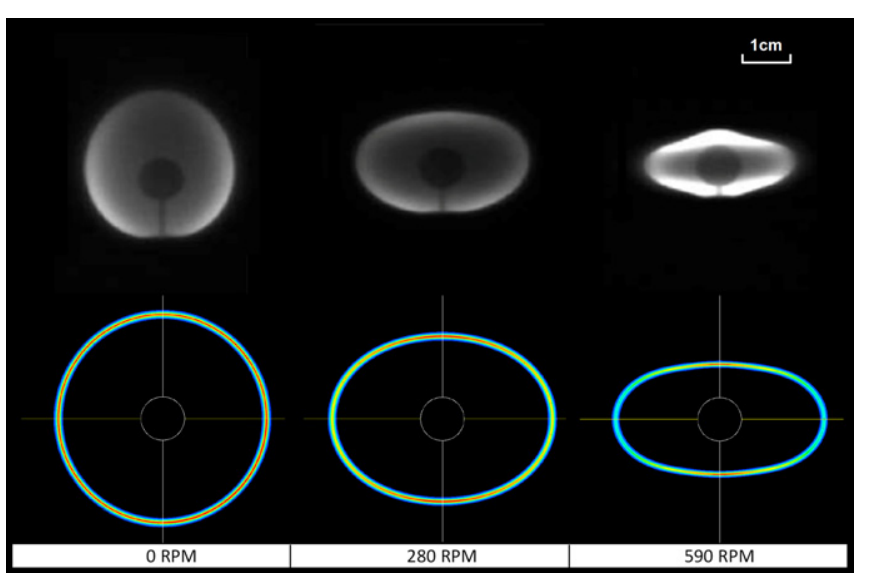

Fig. 8. Experimental and computational flame shapes for case (1) at $\varpi=0,30.1$ and 63.4 .

We shall now examine how the spherical flame responds at such high angular velocities. Specifically, flame distortion at both the poles and the equator will be empirically determined and, if possible, compared with relevant similarity solutions.

\subsubsection{Flame response near the poles}

Figure 6 shows the flame location displacement corresponding to the flame images in Fig. 5a and b, plotted against the angular velocity of the burner. The reference flame radius at no rotation condition for the $m=5.6$ and $8.4 \mathrm{mg} / \mathrm{s}$ are $1.74 \mathrm{~cm}$ and $1.93 \mathrm{~cm}$ respectively. Here and in all the following plots of displacement versus angular velocity, the displacement in the ordinate is normalized by the corresponding non-rotating flame radius while the normalized angular velocity $\varpi$ is the abscissa. It is seen that the normalized flame displacements for two cases nearly collapse on one curve, and has an inflection point around $\varpi=20$. In particular, the displacements seem to scale as $\varpi^{a}$, where $a$ is greater and smaller than unity for small and large angular velocities, respectively. Indeed, when these data are fitted in a log-log plot, the exponent $a$ is determined to be 2.2 and 1.7 at small angular velocities (first four data points), whereas it becomes 0.72 and 0.73 at high angular velocities (last four data points) for $m=5.6$ and $8.4 \mathrm{mg} / \mathrm{s}$, respectively.

The above results indicate that the displacement scales approximately as $\varpi^{2}$ for small angular velocities, in accordance with the analysis of the perturbation theory. For large angular velocities, we note that the flow around the pole can be approximated to that of an infinitely large spinning disk [9] with blowing from its surface. Von Karman [9] first solved the infinite spinning disk problem by recognizing that all variables depend only on the dimension perpendicular to the disk, thereby simplifying the governing equations to an ordinary differential equation. It was subsequently shown that the Von Karman similarity solution still applies when the problem is generalized to include surface suction or injection $[10,11]$. The analysis shows that the induced velocity infinitely away from the disk that brings the flow towards the rotating disk varies with $\omega^{1 / 2}$ when the blowing is small compared to the rotation rate of the disk [12], and is proportional to $\omega^{2 / 3}$ when it is large [12]. Considering the fact that the flame displacement at the poles is due to the entrainment induced by the rotating sphere, we would expect the flame displacement to scale similarly as the induced flow caused by the infinitely large disk and given all the additional complexities induced by flame presence, the exponent $a=0.72-0.73$ is quite close to the above mentioned limiting conditions.

\subsubsection{Flame response near the equator}

The location of the flame displacement around the equator for case (2) is plotted against the angular velocity of the burner in Fig. 7. Similar as the result at the poles, the displacement overall increases with increasing mass flow rate, and scales roughly as $\varpi^{1.5}$ at small angular velocities. However, at very high angular velocities, the flame displacement decreases with further increase in the angular velocity. Furthermore, the onset of the reduction is delayed with increasing mass flow rate. For example, when the mass flow rate is $11.2 \mathrm{mg} / \mathrm{s}$, which was shown in case (2) of Fig. 2, the flame location at the equator does not decrease even at $1690 \mathrm{rpm}$ or $\varpi=85$. We next attempt to explain this nonmonotonic behavior by examining results obtained from computational simulation.

\subsubsection{Results from computations and explanation of the nonmonotonic behavior}

Results from the computation are presented for case (1). Fig. 8 compares the experimental flame shape and the computed reaction rate contours. Since the problem is diffusion controlled, and the diffusive nature of the flow is computationally described quite well, the overall flame shape is correspondingly simulated well, to be discussed next.

Figure 9a and b compares the experimental and computational normalized deviations in the flame location for the pole and equator flame segments, respectively. We first note that, in terms of quantitative predictability, the computed location of the maximum reaction rate $(2.25 \mathrm{~cm})$ for the stationary case deviates from the corresponding experimental location of the flame surface $(1.69 \mathrm{~cm})$ by about only $33 \%$, which can be considered to be quite satisfactory recognizing the sensitive nature of the flame location to the prescription of the transport coefficients [13]. In terms of trend, the computation also simulates well the experimental results at both the pole and equator regions, particularly the nonmonotonic response of the equatorial flame at high angular velocities.

We next show in Fig. 10 the calculated profiles of the velocity and combustion scalars of the flame along the equatorial radius for the three angular velocities $\omega=0,280$ and $590 \mathrm{rpm}$, which correspond to $\varpi=0,30.1$ and 63.4 respectively. We first consider the radial velocity profiles. It is seen that for the non-rotating case the velocity near the burner surface is controlled by that of the ejection velocity of the oxidizer gas, and hence decreases with increasing radial distance. In the presence of rotation, the entrained flow from the polar region adds substantial mass flux to the radial flow, causing the subsequent increase in the radial velocity. The effect is particularly prominent for the $\varpi=63.4$, in which the effect of the surface injection is rapidly diminished by this entrained flow.

Figure 11 shows the streamlines drawn from the tangency condition of velocity vectors in the $r-z$ plane, superimposed with the flame temperature contours for the previous two rotating cases of $\varpi=30.1$ and 63.4 , shown in split frames on left and right of the vertical axis of rotation, respectively. It is seen that while there is not much diversion of polar flow towards the equator for the $\varpi=30.1$ case, for the $\varpi=63.4$ case the streamlines clearly cross the flame, indicating strong entrainment of the polar flow and the accompanying species towards the equatorial region.

Having identified the flow direction in the flame region, we can now explain the nonmonotonic response of the radial flame location with increasing spinning velocity. That is, the inwardly-directed flow in the polar region convects the ambient inert as well as the flame-generated products toward the near equatorial flame location. This then dilutes the oxidizer concentration of the burner mixture as it approaches the radial segment of the flame, causing it to be more conducive to be located closer to the burner in order to maintain stoichiometric burning. Consequently, while the radial 

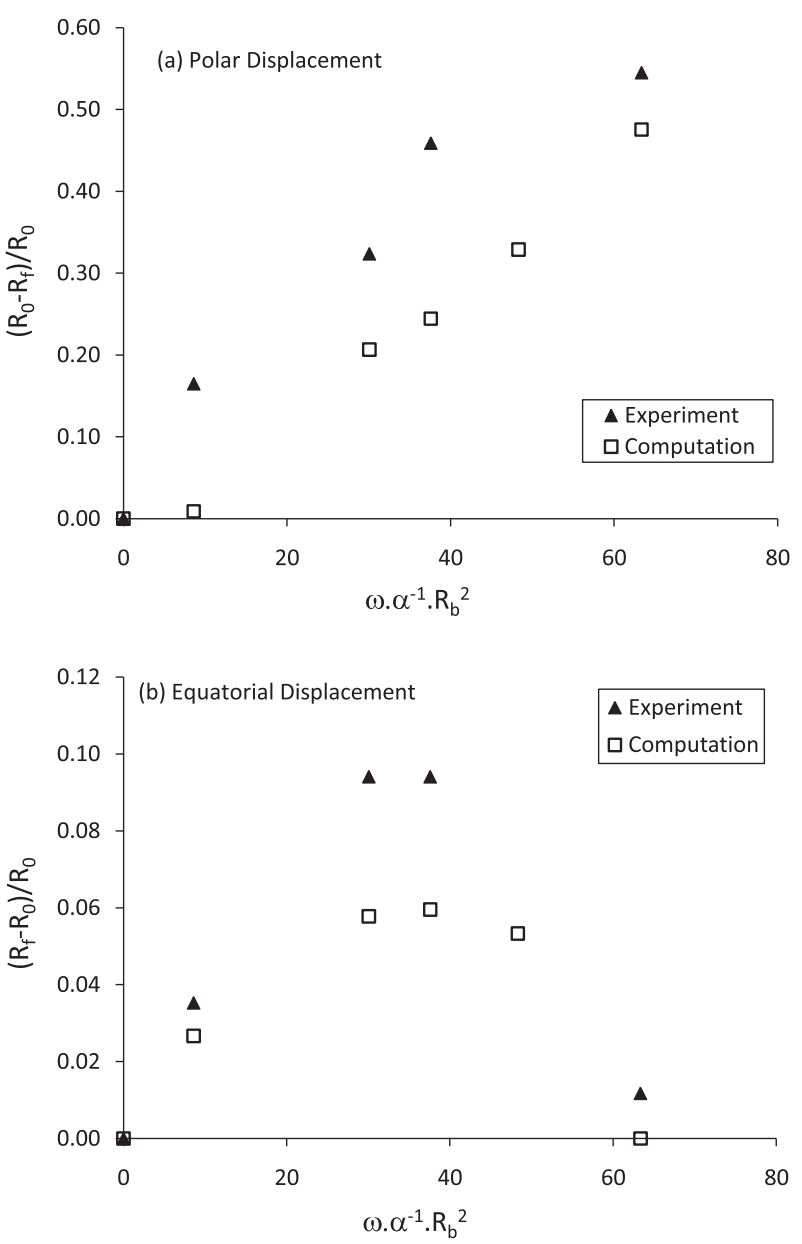

Fig. 9. Comparison of experimental and computational flame displacement (a) at poles (b) at equator, for case (1).

flame tends to move outward for smaller spinning velocities, the dilution effect causes it to retract at higher spinning velocities.

It is also of interest to assess the role of the inert, as compared to that of the combustion product, in inducing this nonmonotonic response. Additional simulations without Ar, while maintaining the fuel mass fraction at unity in the ambient, again revealed the nonmonotonic behavior, suggesting the essential role of the products in diluting the oxidizer concentration and inducing the retraction of the radial flame segment.

\section{Conclusions}

The response of spherical diffusion flames under rotation was studied experimentally in microgravity. When subjected to rotation, the flame deformed into a pancake shape, flattened at the poles and protruded outward at the equator, regardless of the Lewis numbers of the fuel and the oxidizer. The local flame temperature, however, was noticeably influenced by the Le of the system. When the Le of the fuel as burner mixture was less than unity, the light emission at the poles significantly became stronger as rotation increased due to non-equidiffusive effects of changing curvature on the fuel side. When this fuel mixture was switched to the ambient, just the opposite was observed. Finally, the extent of the flame shape deformation was shown to increase linearly with the burner mass flow rate. These observations are in accordance with the results of previous theoretical work [4], even though the

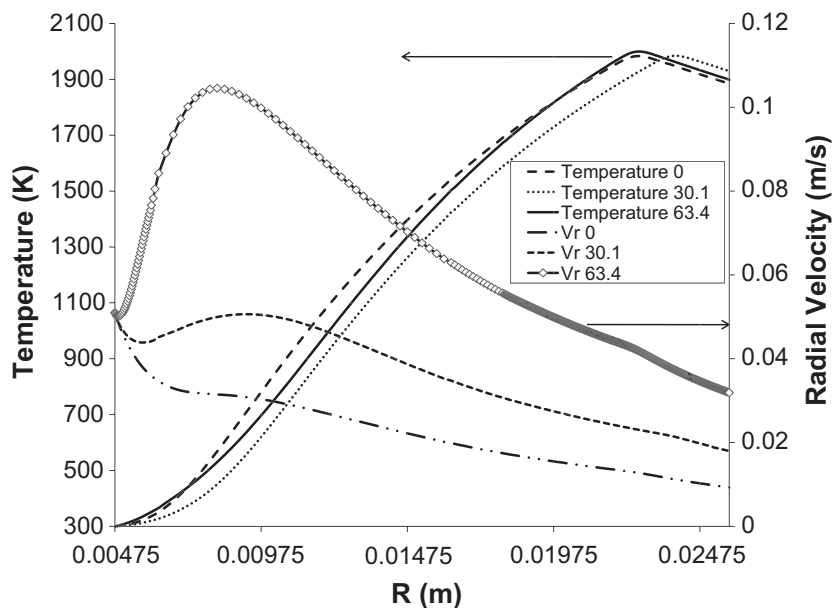

Fig. 10. Plot of temperature and radial velocity versus equatorial radius at $\varpi=0,30.1$ and 63.4

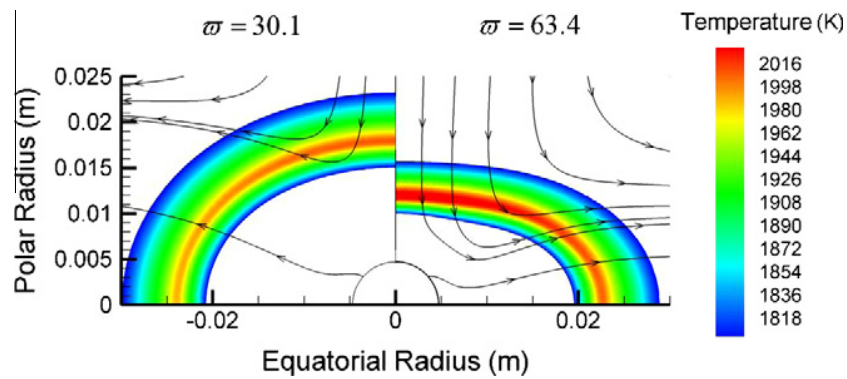

Fig. 11. Streamlines superimposed with temperature contours for $\varpi=30.1$ (left) and $\varpi=63.4$ (right).

theory assumes small rotational perturbations which are contrary to the large rotational velocities in the experiments.

The polar displacement of the flame as a function of the angular velocity was found to scale similarly as that established by an infinitely large spinning disk. The equatorial flame displacement however varied nonmonotonically with increasing spinning velocity, caused by the dilution of the burner oxidizer flow through advection of the products and inerts generated in the polar flame region. This flame contraction phenomenon, due to product and inert enrichment from upstream flame segments could be a general phenomenon associated with flames involving entrainments and evolutionary development, and merits further study.

\section{Acknowledgments}

This work was supported by the NASA Microgravity Combustion Program and a NASA GSRP fellowship program to S.W.Y. The authors would like to acknowledge the assistance of Dr. P. Ferkul, as well as all personnel at the 2.2-second drop tower and Zero-G facility at NASA Glenn Research Center.

\section{References}

[1] H.G. Pearlman, S.H. Sohrab, Combust. Sci. Technol. 76 (1991) 321-334.

[2] D. Lozinski, M. Matalon, Proc. Combust. Inst. 24 (1992) 1483-1491.

[3] D. Lozinski, M. Matalon, Combust. Sci. Technol. 96 (1994) 345-367.

[4] S.W. Yoo, J. Qian, J.K. Bechtold, C.K. Law, Combust. Theor. Model. 9 (2005) 199217.

[5] J. Qian, J.K. Bechtold, C.K. Law, Combust. Flame 110 (1997) 78-91.

[6] S.W. Yoo, On the Structure and Dynamics of Stationary and Rotating Spherical Diffusion Flames, PhD Thesis, Princeton University, 2006. 
7] S.W. Yoo, D.L. Zhu, C.K. Law, Rev. Sci. Instrum. 77 (2006) 075102

[8] C.K. Law, Combustion Physics, Cambridge University Press, 2006.

[9] T. Von Karman, Z. Angew. Math. Mech. 1 (1921) 233-252.

[10] J.T. Stuart, Quart. J. Mech. Appl. Math. 7 (1954) 446-457.
[11] H.K. Kukiken, J. Fluid Mech. 47 (1971) 789-798.

[12] L.A. Dorfman, Hydrodynamic Resistance and the Heat Loss of Rotating Solids, Oliver \& Boyd, 1963.

[13] C.K. Law, H.K. Law, Combust. Sci. Technol. 12 (4) (1976) 207-216. 